\title{
Managing depression in older people
}

\section{Cornelius Katona}

Although common and disabling, depression in old age remains both underdetected and undertreated. This is particularly regrettable in view of its high risk of chronicity, recurrence and suicide, as well as the considerable burden of associated health care costs. Despite the availability of a variety of physical and psychological treatment options of well-established efficacy, there is little recent evidence of improvement in either clinical practice or patient outcome. An understanding of the principles of management of depression in elderly patients requires some knowledge of the differences between such patients and their younger counterparts. The clinical presentation and diagnosis of depression in old age, its epidemiology and prognosis are reviewed briefly below, before focusing on the practicalities of treatment.

The importance of improving detection and management of depression in old age (particularly in the primary care setting) has recently been highlighted by the publication, under the auspices of the Defeat Depression Campaign, of a consensus

Box 1. Consensus statement on recognition and management of depression in late life in general practice

Depression is harder to diagnose in old age

Its prevalence is higher in GP surgery attenders than in community samples

Risk is increased by poor health and life events

Ageist attitudes may impede appropriate management

Physical and psychological treatments are as effective as earlier in life

Depression in old age is a relapsing condition and must be monitored closely statement on recognition and management of depression in late life in general practice (Katona et al, 1995). The key points of the statement are summarised in Box 1.

\section{Assessing depression in older people}

Depression may present in less obvious ways in older people, probably reflecting a combination of cultural factors, pathoplastic effects of ageing and differences between birth cohorts. This provides some explanation for its underdetection, although failure by patients to acknowledge their own depression (or to be aware of its potential for treatment) may also be important. Presentations with prominent lethargy or hypochondriacal complaints are common. 'Neurotic' complaints such as generalised anxiety, subjective nervousness and irritability, may also reflect underlying depression. The distraught, 'importuning' elderly depressed patient may present a particularly difficult challenge to the tolerance as well as the diagnostic skills of the clinician. Formal comparisons of symptom pattern between older and younger patients with established diagnoses of depression show the former to have greater initial insomnia, agitation and hypochondriasis but less depersonalisation, suicidal intent and loss of libido. Crucially, depressed mood is frequently not reported by elderly people who otherwise have all the clinical features of depression. This may be as a result of alexithymia (the inability of patients to verbalise or fantasise affective experience) or of rigid defense mechanisms. A further diagnostic trap is presented by the pattern of 'affective flattening', characterised by unchanging facial expressions, reduced motility, lack of eye contact

Cornelius Katona, FRCPsych, Professor of Psychiatry of the Elderly, University College London Medical School, Wolfson Building, Middlesex Site, Riding House Street, London W1N 8AA. Phone: (44)-171-380-9460; Fax: (44)-171-323-1459. His main research interest is in depression in old age, particularly in primary care. He is Secretary of the College Public Policy Committee. 
Box 2. Differences in clinical features of depression between older and younger people

More common in old age

Insomnia

Agitation

Hypochondriasis

Lethargy

Irritability

Less common in old age

Depressed mood

Depersonalisation

Overt suicidal ideation

or expressive gestures, loss of emotional reactivity, and monotonal voice. This presentation is particularly characteristic of depression in older people in residential care and in those with severe coexistent physical illness - both representing circumstances associated with increased risk of developing depression. The most important differences in presentation between older and younger depressed patients are summarised in Box 2.

An issue of particular importance in assessment is the question of suicide risk. Although the absolute numbers of elderly suicides in the UK are relatively low and the suicide rates in young men have recently risen dramatically, the highest suicide rates are still in men aged over 65 . The relationship between depression and both suicide and attempted suicide is also closest in old age. Factors known to be associated with suicide in older people are bereavement, increasing social isolation, deteriorating physical health, and pain. Any deliberate act of self-harm in an older person should be taken seriously, with particular attention to the exclusion or treatment of underlying depressive illness.

\section{Is depression in old age amenable to screening?}

Since depression in old age is both common and (see below) eminently treatable it is an ideal target for screening and, in particular, for attention within the mandatory over- 75 health check that now forms part of the GP contract. Screening instruments designed for use in younger subjects, like the Montgomery-Åsberg Depression Rating Scale (MADRS; Montgomery \& Åsberg, 1979) or the Zung Depression Scale (Zung, 1965) may be used, although these have received relatively little formal validation in older subjects. A number of screening questionnaires for depression have been designed for and validated in elderly subjects. One of these, the Geriatric Depression Scale (GDS; Yesavage et al, 1983) has not only been extensively validated but appears to retain its validity in the physically ill elderly. It is quick to administer and highly acceptable to patients and has been recommended for hospital use by the Royal College of Physicians and the British Geriatrics Society and in primary care by the Royal College of General Practitioners. It exists in $\mathbf{3 0}$ and 15 item versions. The original 30 item version (with the 15 item version in bold) is shown in the appendix.

Screening instruments like the GDS are undoubtedly of potential use in avoiding underdetection and undertreatment of depression in old age. It must however be borne in mind that screening alone probably has little or no effect in improving subsequent GP behaviour or patient outcome. Iliffe et al (1994) have shown that depression screening in older primary care patients did not have a significant impact either on GP behaviour or on individual patient outcome. The utility of screening would be enhanced enormously by increased public awareness of its potential for treatment and the more widespread adoption of accepted principles for its management. A summary of such principles is provided in the recent consensus statement on depression in old age (Katona et al, 1995).

\section{Subtypes of depression in old age}

Conventional diagnostic subtypings of depression, such as 'endogenous/non-endogenous' or 'severe/ mild' appear less appropriate in elderly depressed patients. A much higher proportion of older patients are classified as 'endogenous' than would be the case for depression earlier in adult life and agreement between diagnostic systems over the endogenous/non-endogenous distinction is poor in older depressed subjects. The severe/mild distinction may also be less informative in old age. In a large community survey, subjects fulfilling criteria for dysthymia (chronic mild depression) according to DSM-III (American Psychiatric Association, 1980) had HDRS scores very similar to those with 'major' depression. A classification of depression in old age based on age at first onset may be more informative (Alexopoulos, 1989). Subjects becoming depressed for the first time in late life are less likely to have first degree relatives with depression. In addition, late first onset has been reported to be associated with more frequent 
cognitive impairment, lower treatment response, a higher rate of brain imaging abnormalities, greater vulnerability to subsequent development of dementia and greater mortality.

\section{Prevalence}

Estimates of the prevalence of depression in elderly people have varied widely, reflecting differences in sample selection, screening instruments and caseness criteria. In particular, studies using criteria designed for and field-tested in younger subjects usually report high levels of depressive symptomatology in old age, but very low prevalence rates for major depression. These anomalous results have encouraged the development of diagnostic interviews and diagnostic algorithms specifically for the elderly, of which the most widely used is the Geriatric Mental Status Schedule (GMS; Copeland et al, 1986). Studies using the GMS are much more consistent and suggest a community prevalence (for depression sufficiently severe not to be out of place in a psychiatric out-patient clinic) of about $15 \%$ (Katona, 1994a). As in younger subjects, the prevalence of depression is higher (by a factor of 1.5 ) in women than in men. Some studies suggest increasing prevalence with increasing age, but this probably reflects concomitant physical illness (discussed below) rather than a direct age effect.

Prevalence rates drawn from community samples are likely to underestimate the scale of the problem since there is consistent evidence for much higher rates in institutional care. Depression is particularly likely to be missed in an institutional setting in which apathy, agitation and irritability are liable to be misinterpreted as part of a dementing illness.

Elderly primary care attenders also appear to have higher prevalence rates for depression (Macdonald, 1986), suggesting that frequent surgery visits (usually with predominantly physical symptomatology) are a characteristic behaviour pattern in elderly people with depression.

\section{Depression and physical illness}

Studies in community, primary care and general hospital samples all suggest that depression is about twice as common in physically ill than in healthy elderly subjects. The main predictors of depression in the physically ill elderly appear to be positive psychiatric history and severity of the physical illness itself.
Depression in elderly medical patients may have an adverse effect on physical prognosis. Associations have been reported with increased length of admission, poor rehabilitation, increased risk of transfer to residential care and increased mortality. Coexistent depression may also distort elderly patients' attitudes to life-sustaining medical therapy, and its presence should always be excluded in patients refusing such treatment.

Depression in elderly medical patients is frequently missed. Rapp et al (1988) found that fewer than $10 \%$ of a series of depressed medical in-patients were correctly recognised by house physicians. Screening questionnaires such as the GDS may be particularly useful in medical settings.

The management of depression in physically ill elderly patients is particularly difficult. Tricyclics are frequently contraindicated and, where used, are associated with particularly high side-effect rates. Clinical trials have been few and small.

\section{Depression in people with dementia}

Wragg \& Jeste (1989) have reviewed several studies examining depressive symptoms and signs in people with established Alzheimer's disease; they reported a median prevalence for depressed mood of $41 \%$ and for depressive disorder of $19 \%$. Most studies suggested a higher prevalence of depression than in non-demented controls. Depression may present prodromally as well as in established dementia. Severe depression may also present with a dementia-like picture (depressive pseudodementia) initially responsive to treatment but with a high subsequent risk of true dementia. Depression may be difficult to assess in patients with severe dementia because of their loss of verbal skills. A number of rating scales, such as the Cornell Scale (Alexopoulos et al, 1988) have been devised, utilising informant history to supplement patient interview and clinical observation. Depression is clinically important in established dementia since it may result in increased behavioural disturbance and loss of daily living skills. Antidepressant treatment in such patients may improve cognitive function as well as depressive symptoms.

\section{Prognosis}

Depression in old age is associated with high chronicity and a considerable risk of relapse even after recovery. Cole (1990) has systematically reviewed 10 follow-up studies, the combined results showing that although $60 \%$ remained well 
or had relapses with recovery, about $25 \%$ developed chronic depression. Depressed elderly patients have a mortality rate approximately double that found in age and sex matched community subjects. At least some of the excess appears to be related directly to the depression since it remains apparent after allowance is made for concurrent physical illness (Murphy et al, 1988).

Several factors have been identified as of prognostic significance within depression in old age. Notable among these are the negative effects of poor physical health and the positive ones of appropriate treatment.

\section{General principles of management of depression in old age}

Depression as identified in community surveys is hardly ever treated. Community studies have reported that as few as $4 \%$ of depressed patients receive appropriate treatment (Copeland et al, 1992). Treatment is almost as unlikely to occur in depressed GP attenders, with rates as low as $10 \%$ reported (Macdonald, 1986) even though the GPs are almost invariably able to recognise their patients' depression on direct questioning.

The first challenge is therefore to break down attitudes in patient and doctor alike which mitigate against treatment being instituted. These include lack of public awareness that depression, even in old age, is a distinct and treatable illness. 'Ageist' medical beliefs to be addressed include the notions that misery is a legitimate response to becoming old, that older people are mentally too rigid to benefit from psychological intervention, and that drugs are too hazardous for 'frail' elderly people.

All the treatment options relevant to depression earlier in life remain effective in old age and, as in younger people, may be more effective combined than in isolation. Drug treatments are most widely available and are most frequently used as 'first line' management, particularly in primary care. Their use should not, however, preclude consideration of psychological treatments, particularly cognitivebehavioural therapy (CBT) and ECT.

\section{Drug treatment}

Although the same principles of antidepressant drug treatment apply as for younger patients, several particular problems (relating mainly to agerelated changes in drug handling and in cognitive function) need to be considered when planning the treatment of individual older patients.

Antidepressant drugs currently in use include 'older' drugs such as the tricyclic antidepressants (TCAs), the related heterocyclic antidepressants (mianserin, maprotiline) and the monoamineoxidase inhibitors (MAOIs); a relatively newly introduced TCA (lofepramine); the selective serotonin reuptake inhibitors (SSRIs); and a novel MAOI, moclobemide. The evidence regarding the efficacy and tolerability of these groups will be discussed, with particular emphasis on the question of whether the advantages of the newer drugs in elderly patients might be sufficient to outweigh their higher cost.

\section{Efficacy}

Controlled antidepressant trials in the elderly published up to 1986 have been reviewed by Gerson et al (1988) and those between 1987 and 1992 by Anstey \& Brodaty (1995). TCAs such as amitriptyline and clomipramine and the heterocyclic antidepressants such as mianserin and trazodone have established efficacy in older patients as does the MAOI phenelzine. Similar efficacy is also evident for SSRIs and for moclobemide. Placebo controlled evidence is relatively sparse, although some evidence is available for a number of TCAs (nortriptyline, imipramine, amitriptyline), for the MAOI iproniazid, and for the SSRIs citalopram, fluvoxamine and fluoxetine. Overall, however, all antidepressant drugs formally evaluated in the elderly seem effective in $50-60 \%$ of patients with, in particular, no clear difference in efficacy between 'older' and 'newer' drugs.

Caution must be used in extrapolating the results of such trials to routine practice, since trials only include patients with relatively severe depression (sufficient to warrant a diagnosis of major depression) who are also physically fit enough to be free of contraindications. Virtually no antidepressant trials have examined very old (aged $80+$ ) samples since such patients, while remaining an important treatment target in clinical practice, almost invariably fulfil one or more of the exclusion criteria for drug trials. Clinical experience suggests however that antidepressant efficacy is similar to that in clinical trial populations.

\section{Tolerability}

Elderly patients are particularly likely to suffer side-effects when taking TCAs or MAOIs and are more vulnerable to serious adverse consequences as a result. The most important of these side-effects are shown in Box 3. Perhaps most crucial is the fact that elderly patients are more vulnerable to 
Box 3. Side-effects of tricyclic antidepressants of particular relevance in older patients

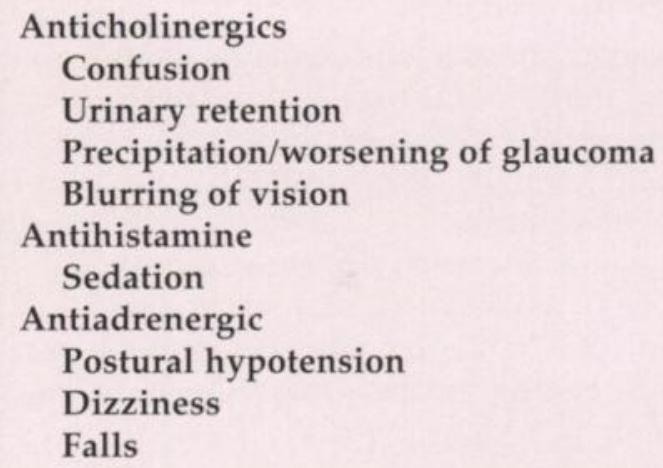

accidents, particularly to falls, which in turn carry an increased risk of osteoporosis-related long bone fracture. This relates mainly to the drugs' potency in blocking alpha-1 adrenergic receptors, thereby exacerbating postural hypotension, which is often present anyway in old age. The antihistaminic effect of most TCAs renders them sedating which may be helpful in agitated depression but may worsen cognitive function and further increase vulnerability to falls and accidents.

Age-related decreases in protein synthesis can increase free plasma TCA levels, as may reduced creatinine clearance and hepatic blood flow. Interindividual variability in blood levels is, however, considerable in elderly as well as younger subjects and (with the exception of nortriptyline which has a well-established optimal therapeutic range) titration to maximum tolerated dose may be as necessary in elderly as in younger subjects, though the average dose required will be smaller.

It should, however, be remembered that while the above considerations are broadly applicable to TCAs (and also to heterocyclic antidepressants and MAOIs), not all of these drugs have identical sideeffect profiles. Lofepramine for example, has a conventional tricyclic structure but appears in both in-vitro studies and trials in non-elderly subjects to be relatively free of anticholinergic side-effects and to cause significantly less impairment in cognitive functioning. However, the cost of treatment with lofepramine is much higher than that of most other TCAs and approaches the cost of SSRIs.

Age-associated pharmacokinetic and pharmacodynamic changes are, in general, less clinically important for newer antidepressants, most of which can be administered in the same dose to elderly as to younger patients. SSRIs and moclobemide are also relatively free of anticholinergic effects and cardiotoxicity, and cause much less postural hypotension than TCAs and MAOIs. The main side-effects of the SSRIs, as in younger patients, are headache and nausea. The review by Anstey \& Brodaty (1995) examines the overall and adverse effect related withdrawal data from several trials comparing newer antidepressants (mainly SSRIs, but also moclobemide) with TCAs and/or heterocyclic antidepressants in elderly depressed patients. Several of these trials report lower withdrawal rates in the SSRI (or moclobemide) groups, although in most of the studies the differences are quite modest. Anstey \& Brodaty emphasise the methodological limitations of the data as reported in the published trials. 'Fitness to treat' on the basis of datasheet indications is another measure of tolerability which can be applied in more representative populations. A recent study (Mullan et al, 1994) suggests that a higher proportion of elderly patients are fit to take SSRIs than TCAs, reflecting their relatively few contraindications.

\section{Practical aspects of treating older patients with antidepressants}

Compliance with antidepressants is often problematic in old age. This reflects higher vulnerability to troublesome side-effects, particularly in the context of comorbid physical illness and coadministration of other drugs with potential adverse interactions. The onset of therapeutic action may take even longer in the elderly, with useful effects sometimes only emerging in the 7th and 8th week of treatment. Patients unaware of such response delay can hardly be blamed for stopping taking tablets which make them feel worse.

The new generation of antidepressant drugs represent a modest step forward in the treatment of depression in elderly patients. This relates mainly to a reduction in practical problems associated with their use in relatively frail patients which may enable a higher proportion of such patients to be treated effectively (Mullan et al, 1994). The question of whether such superiority is sufficient in cost-benefit terms to justify their use as first-line drugs for depression in old age remains open.

\section{Psychological treatments}

It is widely thought that elderly people are not amenable to psychological treatments because their 'mental set' is too rigid to be amenable to therapeutic change. Controlled trials of cognitive, psychodynamic and behavioural treatments, however, reveal them to have similar magnitudes of effect to those found in younger depressed patients. The 
psychotherapeutic focus may be different to that in younger patients, often concentrating on the losses to which older people are particularly vulnerable (bereavement, physical health, financial security etc.) and on fears of impending death.

The best efficacy evidence is for CBT, which has been shown to be an effective treatment for depression in older subjects in a group setting as well as individually, and may thus be economically viable as well as effective in older people. CBT in old age has also been found superior to less specific therapies such as craft or music groups. Depression in old age is associated with the same pattern of negative cognitions (about self, the world and the future) as earlier in life, and a similar cognitive shift with CBT has also been demonstrated.

\section{Electroconvulsive therapy in old age}

Although widely used in old age, ECT remains a particularly controversial treatment option in this age group because of concerns about its safety and about the validity of consent procedures. There is no denying that ECT is less safe in old age than in younger people, but this comparison is irrelevant: the crucial issue is the safety of ECT compared to other treatments or to leaving depression in old age untreated. The safety profile of ECT in the elderly is in fact surprisingly good. It should, however, be borne in mind that such safety relies on good liaison with the anaesthetist. For example, it may be appropriate to delay the procedure until the patient's physical condition is optimised.

The efficacy of ECT in old age is beyond doubt. Mulsant et al (1991) carried out a meta-analysis of 1025 elderly patients receiving ECT in 14 separate studies and reported a favourable outcome in $83 \%$, with full recovery in $62 \%$. This is at least as good an acute response as can be expected in younger patients. ECT may be effective in a broader range of older than younger depressed patients. In contrast with what would be expected earlier in life, prominent comorbid anxiety is associated with a good response to ECT.

ECT is a particularly important treatment option in elderly patients unresponsive to antidepressants, and those with very severe illnesses. It may be lifesaving in patients who are acutely suicidal and those who, in the context of depressive retardation or stupor, have stopped eating and drinking. Particular care must be taken to ensure that the consent obtained is properly informed and that patient and relatives' anxieties about the procedure are fully addressed.
Elderly patients treated with ECT appear to have a surprisingly favourable long-term prognosis; one study (Godber et al, 1987) reported $59 \%$ of subjects entirely well at three year follow-up; most of the remainder (29\%) had only mild residual symptoms and only $3 \%$ had either died or developed a dementia. ECT does not in itself provide any protection against depressive relapse except if given monthly as 'maintenance', a procedure seldom used in the elderly because of increased risk of cumulative memory side-effects. The question of prophylactic treatment (reviewed below) should not be forgotten in patients with a good acute response to ECT.

There is less evidence in old age for bilateral electrode placement being more effective (though this may simply reflect a lack of studies) but unilateral electrode placement is, in the elderly, clearly associated with a reduction in the cognitive side-effects (acute confusion and loss of recent memory) to which older people receiving ECT are particularly vulnerable.

\section{Refractory depression in old age}

In patients failing to respond to first line treatment, a full review of the appropriateness and adequacy (in terms of dosage, duration and compliance) of such a treatment trial should be undertaken. Where appropriate, psychological treatments and/or ECT should be considered.

The many drug combination treatments used in younger patients have received little evaluation in elderly subjects in whom they are also more likely to be hazardous. Lithium augmentation and, to a lesser extent, MAOIs have, however, shown promise in open studies of resistant depression in old age. It must however be borne in mind that older people (particularly those with comorbid dementia and/or Parkinson's disease) are particularly vulnerable to lithium neurotoxicity. Where lithium is used, the initial dose should be low (100$200 \mathrm{mg} /$ day) with gradual dosage titration to achieve blood levels within a downward-adjusted therapeutic range $(0.4-0.8 \mathrm{mmol} / \mathrm{l})$.

\section{Continuation treatment}

As discussed above, depression in old age is a relapsing condition and recovered patients should be monitored closely. The clearest evidence to date for the prophylactic efficacy of antidepressants comes from a placebo-controlled comparison between dothiepin and placebo (Old Age Depres- 
sion Interest Group, 1993). Over a two-year period, relapse rate was reduced by a factor of 2.5 in the dothiepin group. Most other antidepressants so far evaluated appear to have similar prophylactic efficacy; these are reviewed by Katona (1994b) and include a placebo-controlled trial of phenelzine and open studies of fluoxetine, doxepin and lithium.

Other treatments may also result in low relapse rates. These include CBT in several of the studies reviewed by Morris \& Morris (1991) and supportive psychotherapy. Ong et al (1987) randomly allocated 20 elderly depressed in-patients at the point of recovery and discharge to attend a psychotherapy support group or to act as controls. In the subsequent 9 months, none of those in the psychotherapy group had to be readmitted, compared with six out of the ten in the control group.

It should be emphasised that the case for continued antidepressants is based on evidence from relatively severe, hospital treated patients and it must also be borne in mind that long-term use of antidepressants can pose problems as physical health deteriorates with increasing age. As far as milder depression responsive to antidepressants is concerned, the conclusion of the consensus statement on recognition and management of depression in late life in general practice (Katona et al, 1995) is that they should continue the treatment at full dose for at least six months with indefinite opportunistic monitoring.

\section{Conclusions}

Despite being both common and life-threatening, depression in old age is hardly ever treated. A variety of treatments (particularly antidepressants, CBT and ECT) have, however, been shown to be effective, despite the particular challenges to be met in treating such a relatively vulnerable group. Specific treatments should be considered within an overall treatment plan rather than used in isolation. Even in apparently treatment-resistant cases, the response to more aggressive treatment approaches can be gratifying and not necessarily associated with excessive hazard. The high risk of recurrence of depression in old age can be significantly reduced by close monitoring and the judicious use of prophylactic treatments.

\section{References}

Alexopoulos, G. S. (1989). Biological abnormalities in late life depression. Journal of Geriatric Psychiatry, 22, 25-34.

-, Abrams, R. C., Young, R. C., et al (1988) Cornell Scale for depression in dementia. Biological Psychiatry, 23, 271-284.

American Psychiatric Association (1980) Diagnostic and Statistical Manual of Mental Disorders (3rd edn) (DSM-III). Washington, DC:APA.

Anstey, K. \& Brodaty, H. (1995) Antidepressants and the elderly: double-blind trials 1987-1992. International Journal of Geriatric Psychiatry, 10, 265-279.

Cole, M. G. (1990) The prognosis of depression in the elderly. Canadian Medical Association Journal, 142, 633-639.

Copeland, J. R. M., Dewey, M. E. \& Griffith-Jones, H. M. (1986) A computerised psychiatric diagnostic system and case nomenclature for elderly subjects: GMS and AGECAT. Psychological Medicine, 16, 89-99.

-, Davidson, I. A., Dewey, M. E., et al (1992) Alzheimer's disease, other dementias, depression and pseudodementia: prevalence, incidence and three-year outcome in Liverpool. British Journal of Psychiatry, 161, 230-239.

Gerson, S. C., Plotkin, D. A. \& Jarvik, L. F. (1988) Antidepressant drug studies 1946 to 1986: empirical evidence for aging patients. Journal of Clinical Psychopharmacology, 8, 311-322.

Godber, C., Rosenvinge, H., Wilkinson, D., et al (1987) Depression in old age: prognosis after ECT. International Journal of Geriatric Psychiatry, 2, 19-24.

Iliffe, S., Mitchley, S., Gould, M., et al (1994) Evaluation of the use of brief screening instruments for dementia, depression and problem drinking among elderly people in general practice. British Journal of General Practice, 388, 503-508.

Katona, C. L. E. (1994a) The epidemiology of depression in old age. In Depression in Old Age (ed. C. L. E. Katona), pp. 30-41. Chichester: John Wiley.

- (1994b) The prognosis of depression in old age. In Depression in Old Age (ed. C. L. E. Katona), pp. 123-140. Chichester: Wiley.

—, Freeling, P., Hinchliffe, K., et al (1995) Recognition and management of depression in late life in general practice: consensus statement. Primary Care Psychiatry, 1, 107-113.

Macdonald, A. J. D. (1986) Do general practitioners "miss" depression in elderly patients? British Medical Journal, 292, 1365-1367.

Montgomery, S. A. \& Åsberg, M. (1979) A new depression scale designed to be sensitive to change. British Journal of Psychiatry, 134, 382-389.

Morris, R. G. \& Morris, L. W. (1991) Cognitive and behavioural approaches with the depressed elderly. International Journal of Geriatric Psychiatry, 6, 407-413.

Mullan, E., Katona, P., D'Ath, P., et al (1994) Screening, detection and management of depression in elderly primary care attenders. 2. Detection and fitness for treatment: a case record study. Family Practice, 11, 267-270.

Mulsant, B. H., Rosen, J., Thornton, J. E., et al (1991) A prospective naturalistic study of electroconvulsive therapy in late-life depression. Journal of Geriatric Psychiatry and Neurology, 4, 313.

Murphy, E., Smith, R., Lindesay, J., et al (1988) Increased mortality rates in late-life depression. British Journal of Psychiatry, 152, 347-353.

Old Age Depression Interest Group (1993) How long should the elderly take antidepressants? A double-blind placebocontrolled study of continuation/prophylaxis therapy with dothiepin. British Journal of Psychiatry, 162, 175-182. 
Ong, Y. K., Martineau, F., Lloyd, C., et al (1987) A support group for the depressed elderly. International Journal of Geriatric Psychiatry, 2, 119-123.

Rapp, S. R., Walsh, D. A., Parisi, S. A., et al (1988) Detecting depression in elderly medical in-patients. Journal of Consulting and Clinical Psychology, 56, 509-513.

Wragg, R. E. \& Jeste, D. V. (1989) Overview of depression and psychosis in Alzheimer's disease. American Journal of Psychiatry, 146, 577-586.

Yesavage, J. A., Brink, T. L., Rose, T. L., et al (1983) Development and validation of a Geriatric Depression Screening Scale: a preliminary report. Journal of Psychiatric Research, 17, 37-49.

Zung, W. W. K. (1965) A self-rating depression scale. Archives of General Psychiatry, 12, 63-70.

\section{Multiple choice questions}

1. Depression in old age is:

a Commoner in men

b An inevitable consequence of ageing

c Associated with physical illness

d Seldom treated in community samples

e Associated with raised mortality

2. In treating older depressed patients:

a Tricyclics must never be used

b ECT is effective even in patients with comorbid anxiety

c Cognitive therapy is ineffective

d Treatment should be stopped as soon as patients improve

e Lithium dosage is usually similar to that in younger patients

3. Characteristic features of depression late in life include:
a Insomnia
b Increased appetite
c Loss of libido
d Agitation
e Irritability

4. The prognosis of depression in old age is:

a Unaffected by treatment

b Associated with a high relapse rate

c Worse in those with physical illness

d Of chronic illness in $50 \%$ of cases

e Usually of progression to dementia

\begin{tabular}{|c|c|c|c|c|c|}
\hline \multicolumn{6}{|c|}{ MCQ answers } \\
\hline 1 & 2 & & 3 & & 4 \\
\hline a $F$ & & F & & $\mathrm{T}$ & a $F$ \\
\hline b $F$ & & $\mathrm{~T}$ & & F & b $\mathrm{T}$ \\
\hline c $\mathrm{T}$ & & F & & F & c $\mathrm{T}$ \\
\hline & d & F & & $\mathbf{T}$ & d F \\
\hline e $T$ & & F & & $\mathrm{T}$ & e $F$ \\
\hline
\end{tabular}

\section{Appendix: The Geriatric Depression Scale}

Score 1 for answer in capitals.

0-10: not depressed; 11-20: mild depression; 2130: severe depression.

15 item version in bold. 0-5: not depressed; 6-15: depressed.

Are you basically satisfied with your life? yes/NO Have you dropped many of your activities and interests?

YES/no

Do you feel that your life is empty? YES/no

Do you often get bored?

YES/no

Are you hopeful about the future? yes/NO

Are you bothered by thoughts you can't

get out of your head

YES/no

Are you in good spirits most of the time? yes/NO

Are you afraid that something bad is

going to happen to you?

YES/no

Do you feel happy most of the time?

yes/NO

Do you often feel helpless?

YES/no

Do you often get restless and fidgety?

YES/no

Do you prefer to stay at home, rather

than going out and doing new things? YES/no

Do you frequently worry about the

future?

YES/no

Do you feel you have more problems

with memory than most?

YES/no

Do you think it is wonderful to be alive now?

yes/NO

Do you often feel downhearted and

blue?

Do you feel pretty worthless the way

you are now?

YES/no

Do you worry a lot about the past?

YES/no

YES/no

Do you find life very exciting?

Is it hard for you to get started on new

projects?

yes/NO

Do you feel full of energy?

YES/no

yes/NO

Do you feel that your situation is

hopeless?

YES/no

Do you think that most people are better off than you are?

YES/no

Do you frequently get upset over little

things?

YES/no

Do you frequently feel like crying? YES/no

Do you have trouble concentrating? YES/no

Do you enjoy getting up in the morning? yes/NO

Do you prefer to avoid social gatherings? YES/no

Is it easy for you to make decisions? yes/NO

Is your mind as clear as it used to be? yes/NO

Reprinted with permission from Journal of Psychiatric Research, Vol. 17, Yesavage/Brink et al. "The Geriatric Depression Scale". 1983, Elsevier Science Ltd., Oxford, UK. 